\title{
Semi-liquid metal and adhesion-selection enabled rolling and transfer (SMART) printing: A general method towards fast fabrication of flexible electronics
}

\author{
Rui Guo ${ }^{1}$, Siyuan $\mathrm{Yao}^{2}$, Xuyang Sun $^{2}$ and Jing Liu ${ }^{1,2^{*}}$
}

\begin{abstract}
Recent breakthrough in eutectic gallium-indium alloy has revealed its great potential in modern electronic engineering. Here, we established a general method towards super-fast fabrication of flexible electronics via semi-liquid metal and adhesion-selection enabled rolling and transfer (SMART) printing on various substrates. Based on the semiliquid metal and its adhesion-difference on specifically designed target materials, we demonstrated that the rolling and transfer printing method could serve to rapidly manufacture a wide variety of complicated patterns with high resolution and large size. The process is much faster than most of the currently existing electronic fabrication strategies including liquid metal printing ever developed, and the cost either in time or consumption rate is rather low. As illustrated, a series of functional flexible and stretchable electronics such as multiple layer and large area circuits were fabricated to show their superior merit in combination with electrical conductivity and deformability. In addition, it was also demonstrated that the electronics fabricated in this way exhibited good repeatablity. A most noteworthy advantage is that all the fabrication processes could be highly automatic in the sense that user-friendly machines can thus be developed. This method paves a practical way for super-fast soft electronics manufacture and is expected to play an important role in the coming industry and consumer electronics.
\end{abstract}

Keywords: Ni-EGaIn, semi-liquid metal, fast fabrication, adhesion difference, flexible electronics

\section{INTRODUCTION}

With large potential to overcome fundamental mismatch in mechanics between human and machines, flexible electronics [1-3] have been adopted in a variety of applications such as health monitoring systems [4,5], human-machine interactions [6-8] and artificial intelligence [9]. One of the most successful methods for building stretchable electronic devices is to link rigid active components with stretchable connectors [10]. At present, integrating inorganic or organic electronic materials with elastomeric substrates is the major approach to fabricate stretchable conductors. However, complicated and expensive fabrication and limited stretchability hinder the application of inorganic electronic materials [11-15]. The organic electronic materials own good stretchability, but their conductivities are lower than those of metal materials [16-19].

Recently, eutectic gallium-indium alloy (gallium-based liquid metal alloy, EGaIn) exhibits favorable properties such as excellent liquidity [20], high electro-conductivity (EGaIn: $3.4 \times 10^{6} \mathrm{~S} \mathrm{~m}^{-1}$ ) [21], non-toxicity [22-24], zero stiffness and fantastic stretchability [25], which has shown potential application in flexible electronics. By taking full advantage of the deformability of EGaIn, various flexible electronic devices have been fabricated, including triboelectric nanogenerators [26], stretchable wireless power transfers [27], stretchable electromagnetic actuators [28] and stretchable loudspeakers [29]. In addition, various fabrication methods have been developed to pattern the liquid metals on flexible substrates. For example, stretchable micro-channels filled with liquid metals can be employed for flexible interconnects [30] and antenna. This micro-channel injection method needs to prepare

\footnotetext{
${ }^{1}$ Department of Biomedical Engineering, School of Medicine, Tsinghua University, Beijing 100084, China

${ }^{2}$ Beijing Key Lab of CryoBiomedical Engineering and Key Lab of Cryogenics, Technical Institute of Physics and Chemistry, Chinese Academy of Sciences, Beijing 100190, China

* Corresponding author (email: jliubme@tsinghua.edu.cn)
} 
$\mathbf{a}$
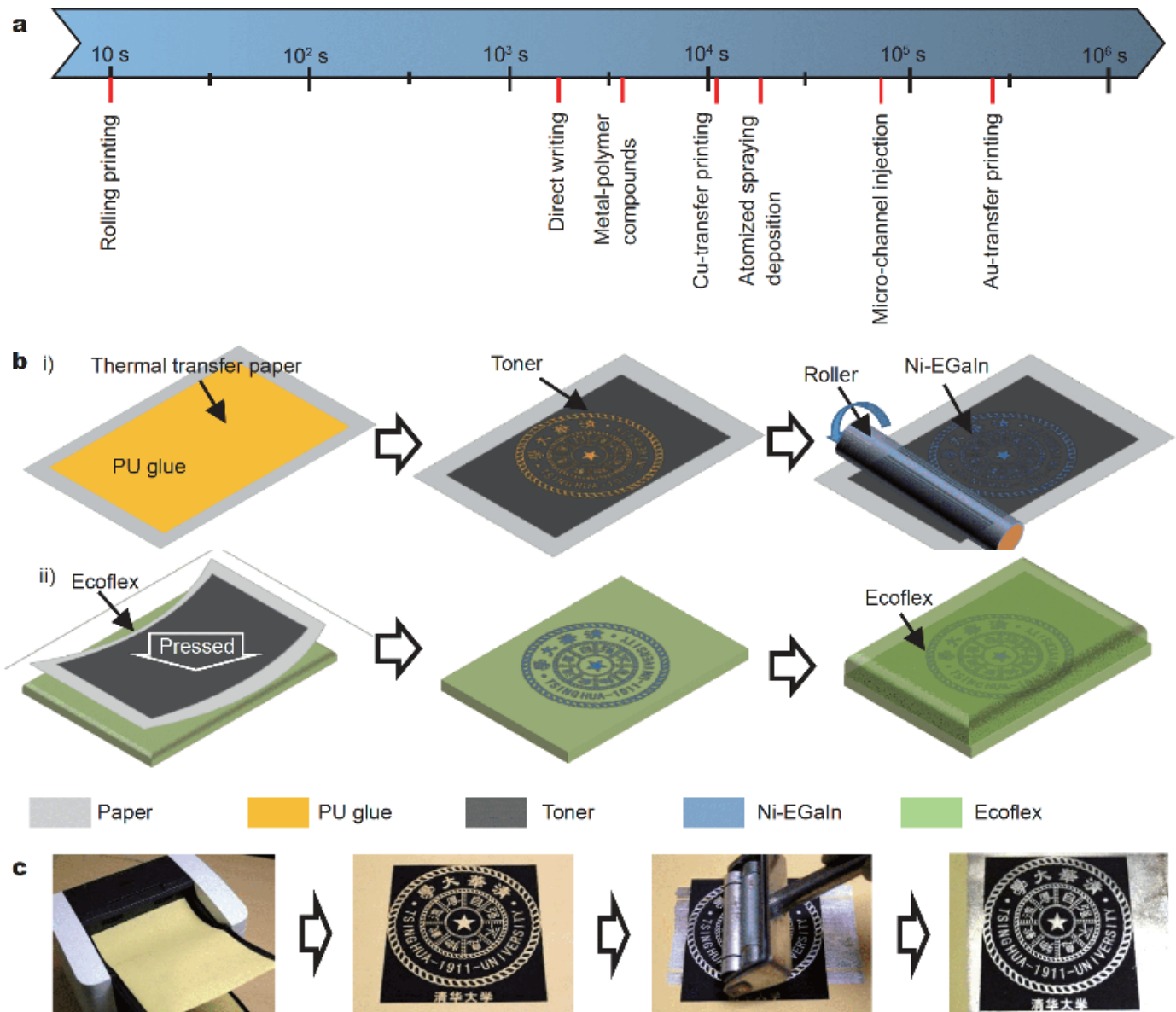

PU glue
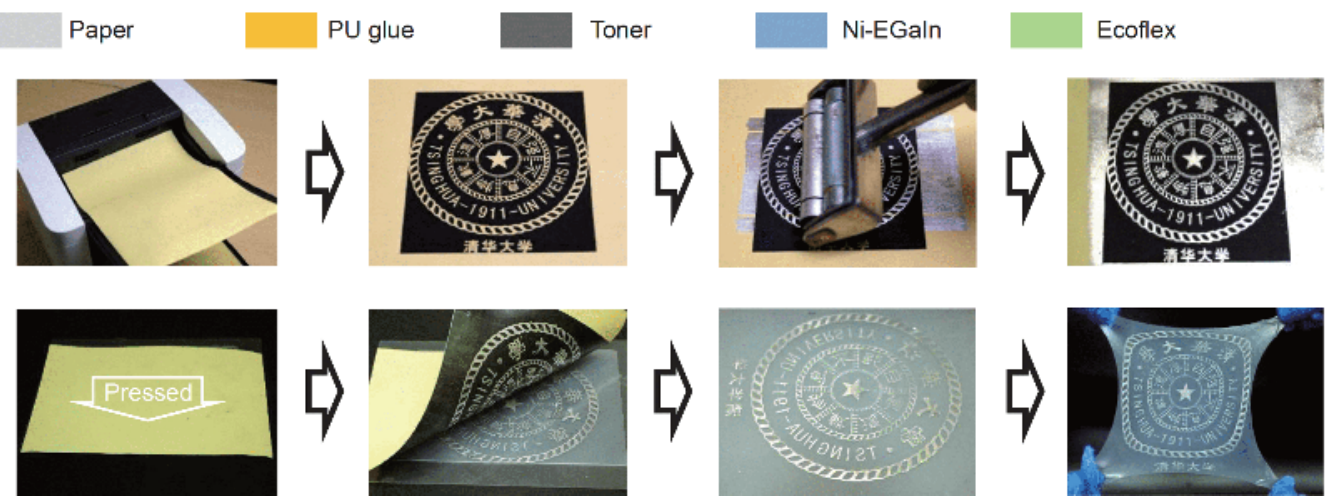

Figure 1 (a) The periods of various methods in preparing flexible electronics. (b) The schematic diagram of semi-liquid metal rolling and transfer printing method. (c) The photographs about the preparation process of rolling and transfer printing method.

microchannel structures using lithography, which is rather complicated (the preparation period is about 3 day) and expensive. Atomized spraying deposition [31-33] is another widely used method for fabricating liquid metal electronics, while the prefabricated masks increase the manufacturing costs and time (the period of mask preparation is about $5 \mathrm{~h}$ ). Besides, direct writing using a micro-syringe is an efficient preparation method to print the liquid metal on elastic substrates directly [3437]. Meanwhile, the complexity of circuits will significantly increase the preparation period (the pattern in Fig. $1 \mathrm{~b}$ takes about $30 \mathrm{~min}$ using the liquid metal printer system). Transfer printing is a one-step preparation method. For example, solid metal $(\mathrm{Cu}, \mathrm{Au})$ can be soaked with liquid metal to fabricate flexible electronics, while the solid metal needs to be printed on elastic substrates using laser engraving [38] (the preparation period of masks is about $3 \mathrm{~h}$ ) or magnetron sputtering [39] (the preparation period of masks is about $20 \mathrm{~h}$ ). In addition, metal-polymer compounds can result in microstructured and conductive path under the mechanical action [40]. However, the preparation of metal-polymer compounds is usually complex, and the mechanical action increases the preparation period due to the complexity of patterns (all the preparation period is about $1 \mathrm{~h}$ ). Fig. 1a shows the preparation periods of different liquid metal printing methods.

In this paper, the semi-liquid metal (Ni-EGaIn) exhibits 
poor adhesion on toners, which could be printed in advance on various flexible substrates using laser printers. According to the adhesion difference of Ni-EGaIn on toners and polyurethane (PU) glue, we developed a super-fast fabrication method for the flexible electronics: semi-liquid metal and adhesion-selection enabled rolling and transfer (SMART) printing. Here, the Ni-EGaIn could be rolling printed on thermal transfer paper in $10 \mathrm{~s}$, which is much faster than that using most of the other circuits manufacturing methods including liquid metal printing methods ever developed so far. More importantly, the Ni-EGaIn printed on paper could also be transfer-printed on ecoflex substrates or other media to fabricate stretchable electronics. A series of paper and stretchable electronics, such as LED array, multilayer circuits and strain sensor were fabricated showing their excellent electrical stability and significant application potential for flexible electronics. Furthermore, we found that the printed inks of Ni-EGaIn with recyclable ability made it possible to further reduce the cost of fabrication and environmental pollution.

\section{EXPERIMENTAL SECTION}

\section{Preparation of Ni-EGaIn}

The EGaIn liquid metal used in this paper is EGaIn composed of $75.5 \mathrm{wt} \%$ gallium and $24.5 \mathrm{wt} \%$ indium (Anhui Minor New Materials Co. LTD, 99.999\% purity), with a low melting point $\left(15.5^{\circ} \mathrm{C}\right)$ and a high electrical conductivity $\left(3.4 \times 10^{6} \mathrm{~S} \mathrm{~m}^{-1}\right)$. Firstly, the gallium $(75.5 \mathrm{~g})$ was put in a beaker, and heated in water bath at $\left(60^{\circ} \mathrm{C}\right)$ for $10 \mathrm{~min}$. After the gallium was melted, the indium $(24.5 \mathrm{~g})$ was further added to the beaker. Then, the Ga-In mixture was kept at $80^{\circ} \mathrm{C}$ for $1 \mathrm{~h}$ with vigorous stirring to mix uniformly. Later, some nickel $(\mathrm{Ni})$ particles (Beijing DK Nano Technology Co., Ltd. diameter of $48 \mu \mathrm{m}$ ) are scattered on the surface of GaIn alloy. In particular, EGaIn was agitated constantly by a glass bar until the $\mathrm{Ni}$ particles were mixed in EGaIn totally (Fig. S1a). Finally, we obtained the GaIn alloy mixed with micro Ni particles, which is called Ni-EGaIn alloy. Here, the gradient content of micro Ni particles was mixed in GaIn alloy (from $0 \%$ to $20 \%)$. The packing ratio is defined as $\phi=m_{\mathrm{Ni}} / m_{\mathrm{GaIn}}$, where $m_{\mathrm{Ni}}$ and $m_{\mathrm{GaIn}}$ represent the mass of the Ni particles and the liquid metal, respectively.

\section{Printing equipment and substrates}

In the present work, the thermal transfer paper (purchased from Shandong HONGTAI PAPER Co., Ltd.) was chosen as the printing substrate, which was covered by a layer of PU glue. The toners were printed on the thermal transfer paper by a laser printer (LJ2205, purchased from Beijing Lenovo Co., Ltd.). Here, The Ecoflex 00-30 precursors (Smooth-On, PA, USA) were mixed with a ratio of 1:1 by volume and stirred evenly. To remove air bubbles, the mixed Ecoflex 00-30 precursors were placed in a desiccator at 36 Torr for $5 \mathrm{~min}$. After $15 \mathrm{~min}$ at room temperature, the mixed Ecoflex 00-30 precursors cured.

\section{Measurements}

In order to identify the microscopic features of the toners, PU glue, Ni-EGaIn and ecoflex, the micrographs were captured with an environmental scanning electron microscope (Quanta 200). The composition of metal oxides on the surface of EGaIn was identified by the Xray photoelectron spectroscopy (PHI Quantera II).

The adhesion of Ni-EGaIn on toners, PU glue and ecoflex were evaluated through four different ways. i) The contact angle of the Ni-EGaIn droplets on the toners, PU glue and Ecoflex, was measured by the contact angle meter (XG-CAMC). ii) The adhesion force of Ni-EGaIn droplets on the toners, PU glue and Ecoflex was tested by the dynamic contact angle measuring instrument (DCAT25, Data Physics Instruments GmbH). Over this test, all the samples (three pieces of paper covered with the toners, PU glue and Ecoflex) were attached to a stress probe, which was connected to a fixed stress sensor. The $\mathrm{Ni}$-EGaIn was held by a container which can be vertically adjusted. The probe was first pushed into the Ni-EGaIn sample and then pulled out at controlled speeds during a complete test. The force acting on the probe was recorded during the whole process. The inserting depth $(D)$ was set as $2 \mathrm{~mm}$ for all the cases. The moving speed of the displacement adjuster was set as $0.05 \mathrm{~mm} \mathrm{~s}^{-1}$ during all the stages except for the stage when the probe reached the sample surface, during which an approaching speed of $0.5 \mathrm{~mm} \mathrm{~s}^{-1}$ was used, as shown in Fig. S3. In this process, the tension was recorded by the dynamometer and the maximal tension was selected as the adhesion force. iii) An obliquity experiment was adopted to assess the adhesive stability of the Ni-EGaIn droplets on three pieces of paper covered with toners, PU glue and Ecoflex, respectively. The photos of the inclined plane where the EGaIn droplets rolled off were recorded with a camera (Canon EOS 800D). iv) The toners were printed on the thermal transfer paper covered with PU glue by laser printers and displayed striated patterns. The Ni-EGaIn was printed on the thermal transfer paper by a brush.

To further investigate the electrical properties of the 
liquid metal printed on the paper, the resistance of $\mathrm{Ni}$ EGaIn lines was measured with Agilent 34420. The electrical conductivities of the Ni-EGaIn samples were measured using a standard four-point method. The samples were filled into a $750 \mathrm{~mm}$ long groove with a rectangular cross section of $5.1( \pm 0.03) \mathrm{mm} \times 4.1( \pm 0.06)$ $\mathrm{mm}$.

\section{RESULTS AND DISCUSSION}

Fig. $1 \mathrm{~b}$ demonstrates the major steps of semi-liquid metal rolling and transfer printing method based on the adhesion-selection of Ni-EGaIn on the toner and PU glue (Supplementary information video S1). The SMART printing method mainly includes two steps: (i) the semiliquid metal rolling printing method on the paper. The thermal transfer paper covered with the PU glue was put into the laser printer, and the predesigned pattern was printed on the thermal transfer paper with the PU glue. Then, the Ni-EGaIn was printed on the paper with the toner pattern by a roller. Due to the significant adhesion difference of Ni-EGaIn on the toners and PU glue, the $\mathrm{Ni}$ EGaIn was selectively printed on the PU glue with the larger adhesion force, other than the surface of toners. (ii) The semi-liquid metal transfer printing method on ecoflex. The Ni-EGaIn printed on the thermal transfer paper could be transfer-printed on the ecoflex substrate ( $1 \mathrm{~mm}$ thick). The side of thermal transfer paper with $\mathrm{Ni}$ EGaIn was put on the surface of ecoflex substrate. To improve the effect of transfer printing, a pressure $(2.5 \mathrm{kPa})$ was applied to the thermal transfer paper to accelerate transfer printing. Afterwards, the thermal transfer paper was removed from the ecoflex substrate, and the Ni-EGaIn would be transfer-printed on the ecoflex directly. Finally, some mixed Ecoflex 00-30 precursors were poured on the transfer-printed Ni-EGaIn to pack the Ni-EGaIn pattern. The mixed Ecoflex 00-30 precursors would cure after $15 \mathrm{~min}$ at room temperature. Fig. 1c shows the photos about the major steps of liquid metal rolling and transfer printing method. Moreover, some electronic components could be integrated with the Ni-EGaIn lines, and the pins of these components bathed into the Ni-EGaIn, which provided the electrical connections to these components.

The semi-liquid state of the Ni-EGaIn is the iconic feature of the materials, which will affect their performance in the applications of flexible electronics. Therefore, the formation mechanism of the semi-liquid state of the Ni-EGaIn was explored firstly. Fig. 2a demonstrates the photographs of Ni-EGaIn with different weight ratios of micro Ni particles. The surface roughness of the Ni-EGaIn is significantly increased with the content of micro Ni particles. The previous studies of GaIn alloy mixed with solid metal particles $[41,42]$ have also suggested that the solid metal particles in EGaIn would reduce their liquidity due to the metal oxides covered on these solid metal particles, which is in agreement with our results. The schematic diagram in Fig. S1b shows the forming process of the semi-liquid metal (Ni-EGaIn). At first, a layer of metal oxides covered on the surface of EGaIn. Then, Ni particles were added into EGaIn, and floated on the surface because of the high surface tension of EGaIn. Through stirring, Ni particles were wrapped by the metal oxides, which made it easy to be swallowed by EGaIn. On the other hand, the metal oxides wrapped around the Ni particles were also brought into EGaIn, which would accelerate the oxidation of EGaIn. Above all, the increase of metal oxides and $\mathrm{Ni}$ particles in the EGaIn resulted in the formation of the semi-liquid state of the Ni-EGaIn.

Generally, the properties of Ni-EGaIn would change with the addition of the micro Ni particles. To achieve the Ni-EGaIn with the adequate conductivity and favorable printability, we printed five different kinds of Ni-EGaIn (gradient contents of micro Ni particles from $0 \%$ to $20 \%$ ) on the PU substrate, as shown in Fig. S1c. The results show that the semi-liquid metal with $\phi$ below $10 \%$ tended to gather into droplets under the gravity when it was printed on other substrates due to the higher surface tension. While, the Ni-EGaIn with $\phi$ above $10 \%$ could be evenly spread out on the PU glue and provide stable electrical connections. Besides, we measured the conductivity of Ni-EGaIn (gradient contents of micro $\mathrm{Ni}$ particles from $0 \%$ to $20 \%$ ), and the results showed that the conductivity decreased gradually with increasing amount of micro Ni particles, as shown in Fig. 2b. Finally, the semi-liquid metal (Ni-EGaIn with $10 \%$ micro Ni particles) was used in the following experiments due to the appropriate conductivity, and adhesion properties.

The previous studies have shown that the Ni-EGaIn surface is covered with gallium oxide layer in air [43,44], and the adhesion between two surfaces is generally dependent on the chemical interaction and the morphological microstructures of the surface [45]. In this paper, we tested the surface composition of the Ni-EGaIn using XPS and the results clearly demonstrated the presence of $\mathrm{Ga}_{2} \mathrm{O}_{3}$ on the surface of the Ni-EGaIn (Fig. S2). In general, the $\mathrm{Ga}_{2} \mathrm{O}_{3}$ has a higher affinity to various substrates than EGaIn [46,47]. The SEM image in Fig. 3a shows that the PU substrate has very smooth surface, where the Ni-EGaIn has good wettability and 


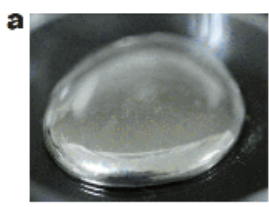

$\phi=0 \%$

b

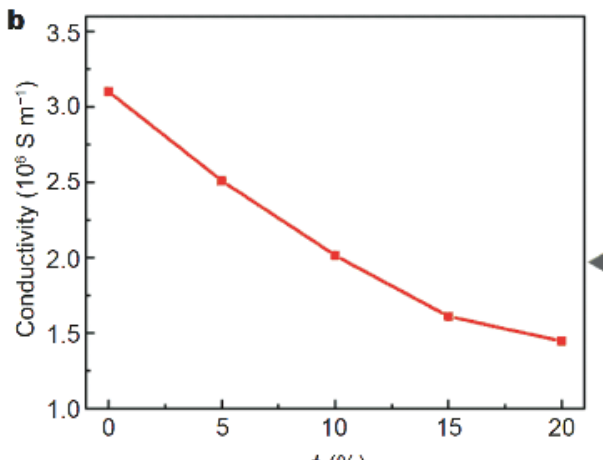

$\phi(\%)$

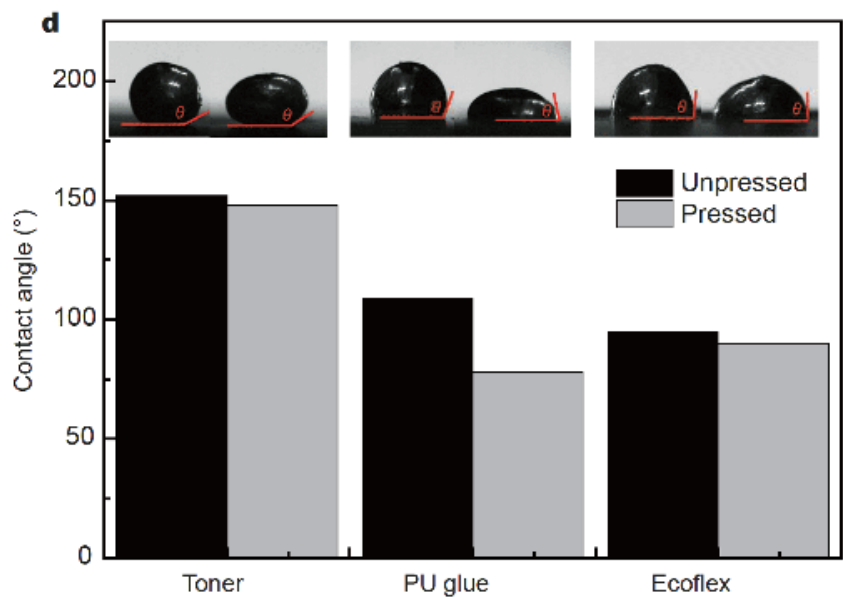

f

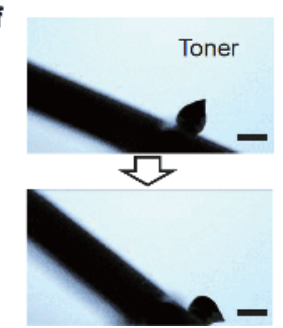

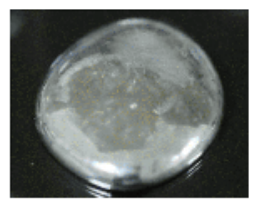

$\phi=5 \%$

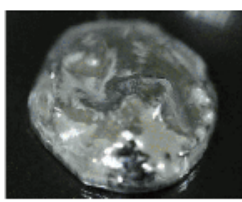

$\phi=10 \%$ c

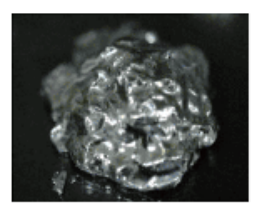

$\phi=15 \%$

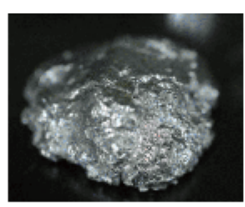

$\phi=20 \%$
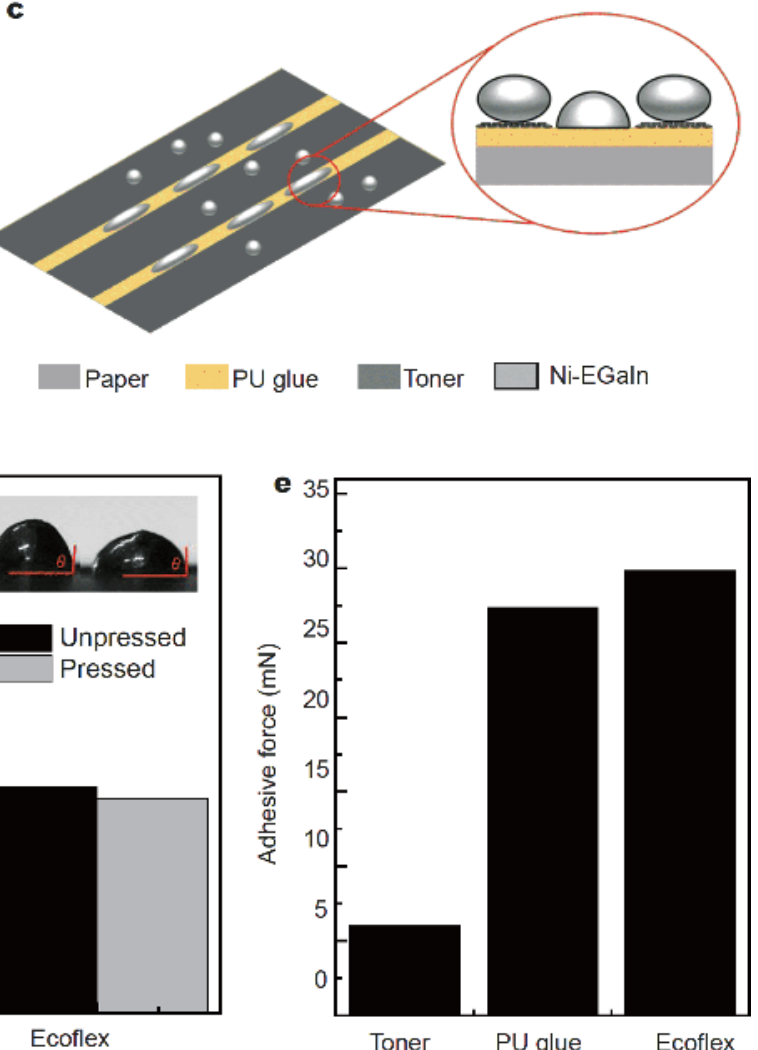

g

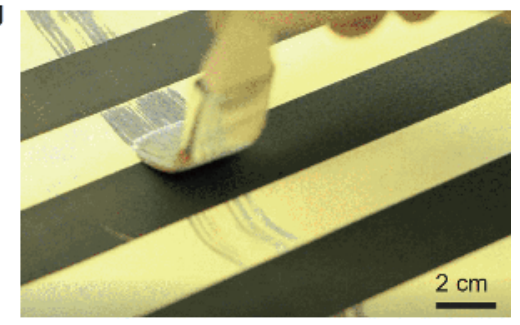

Figure 2 (a) Photographs of Ni-EGaIn with different contents of micro Ni particles. (b) The conductivity of Ni-EGaIn with different contents of micro Ni particles. (c) The schematic diagram about the adhesion mechanism of Ni-EGaIn on PU glue and toners. (d) The contact angle of Ni-EGaIn on toners, PU glue and ecoflex substrates. (e) The adhesion force of Ni-EGaIn on toners, PU glue and ecoflex substrates. (f) The images of Ni-EGaIn on toners, PU glue and ecoflex substrates in the obliquity experiments (scale bar $=2 \mathrm{~mm}$ ). (g) The photograph of Ni-EGaIn printed on the thermal transfer paper by a brush, while not the toners.

adhesion. While, the PU substrate covered with toners has a very rough surface (Fig. 3b), decreasing the contact area between Ni-EGaIn droplets and PU substrates. So it is hard for the Ni-EGaIn droplets to adhere to the substrate, as shown in Fig. 2c. As a result, the Ni-EGaIn could be rolling printed on PU substrate, rather than the toners. Meanwhile, the ecoflex substrate also has very smooth surface (Fig. 3c), and strong adhesion with NiEGaIn, making it possible to transfer-print the Ni-EGaIn on its surface. 


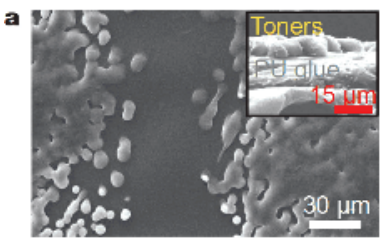

PU glue

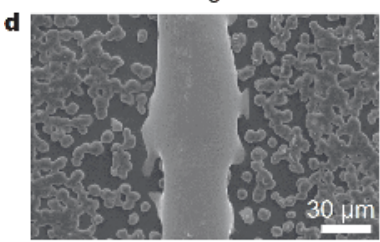

Ni-EGaln on paper

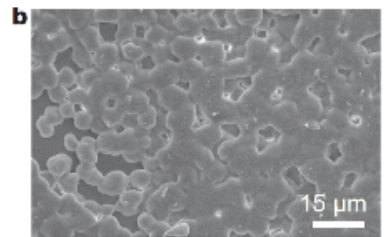

Toner

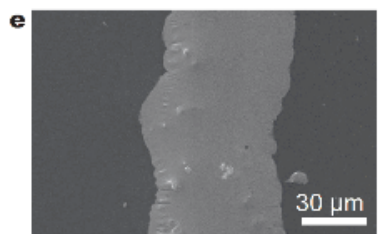

Ni-EGaln on Ecoflex

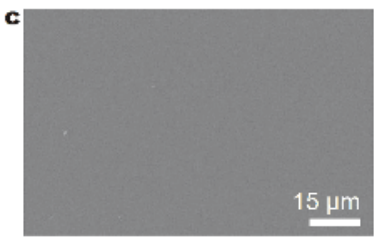

Ecoflex

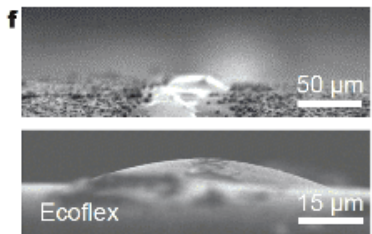

$15 \mu \mathrm{m}$ $\mathbf{g}$

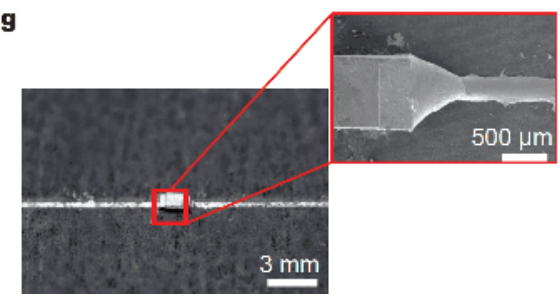

h

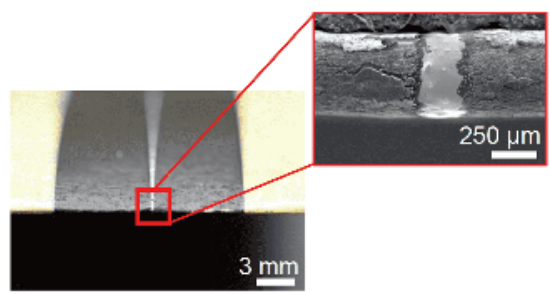

Figure 3 The SEM image of the PU glue and its cross-section image (a), the printed toners on PU glue (b), the ecoflex substrate (c), the Ni-EGaIn line rolling printed on PU glue (d), the Ni-EGaIn line transfer printed on ecoflex (e). (f) The cross-section images of the Ni-EGaIn line printed on PU glue and the Ni-EGaIn transferred on Ecoflex. (g) The photograph and SEM image of the small chip resistor attached with the Ni-EGaIn line. (h) The photograph of folded paper and the SEM image of the bending part showing continuous state of Ni-EGaIn lines.

The results of the contact angle test show the adhesion difference of Ni-EGaIn on the toners, PU glue and ecoflex. As shown in Fig. 2d, the contact angles of the NiEGaIn droplets on toners, PU glue and ecoflex are $152^{\circ}$, $109^{\circ}$ and $95^{\circ}$, respectively. Then, we applied a pressure (about $9.8 \mathrm{mN}$ ) on the Ni-EGaIn droplets using a polytetrafluoroethylene block $(15 \mathrm{~mm} \times 15 \mathrm{~mm} \times 2 \mathrm{~mm}$, weight of $1 \mathrm{~g}$ ). All the contact angles decreased and the contact angles of the Ni-EGaIn droplets on the toners, PU glue and ecoflex were $148^{\circ}, 78^{\circ}$ and $90^{\circ}$, respectively. The smaller contact angles on the PU glue and ecoflex showed improved adhesion with Ni-EGaIn droplets, indicating that the Ni-EGaIn could spread quickly and evenly on the PU glue and ecoflex. Fig. S3a shows the photos of the adhesion force tests, and Fig. S3b presents the schematic illustration of the steps for the adhesion force tests. Fig. S3c shows the tension curves of toners, PU glue and ecoflex substrates with Ni-EGaIn during the push-andpull process. The maximal tension was selected as the adhesion force. In the tests, the adhesion force of $\mathrm{Ni}$ EGaIn on toners $(6.01 \mathrm{mN})$ was obviously weaker than that on the PU glue $(27.35 \mathrm{mN})$ and the ecoflex $(29.84 \mathrm{mN})$, as shown in Fig. 2e. Besides, the obliquity experiment shows that the Ni-EGaIn droplets on toners rolled off from the inclined plane with an inclination of $37^{\circ}$. While the Ni-EGaIn droplets on the PU glue and ecoflex did not roll off from the inclined plane even with an inclination of $90^{\circ}$ due to the strong adhesive force, as shown in Fig. 2f. In Fig. 2g, the Ni-EGaIn could be printed on the PU glue easily by a brush, while there is no $\mathrm{Ni}$-EGaIn on the surface of toners. The adhesion difference of Ni-EGaIn on the toners, PU glue and ecoflex is obvious, as the iconic mechanism of the rolling and transfer printing method.

Furthermore we also investigated the microscopic feature of the printed toners on the PU glue (Fig. 3a) and the Ni-EGaIn lines (Fig. 3d). From these images, the $\mathrm{Ni}$-EGaIn is filled in the gap between the toners on the two sides and adheres to the PU glue. The thickness of PU glue on the paper was about $15 \mu \mathrm{m}$, and that of toners on PU glue is about $8 \mu \mathrm{m}$. At present, the minimum width of $\mathrm{Ni}-\mathrm{EGaIn}$ lines is about $50 \mu \mathrm{m}$, which depends on the gap width of the printed toners. Fig. 3e shows the Ni-EGaIn line transfer-printed on ecoflex, and the minimum width of Ni-EGaIn lines is about $60 \mu \mathrm{m}$, which is slightly wider than that on the PU glue. Fig. 3f shows the cross section micrographs of the Ni-EGaIn line printed on PU glue and the Ni-EGaIn transferred on ecoflex. In addition, a small 

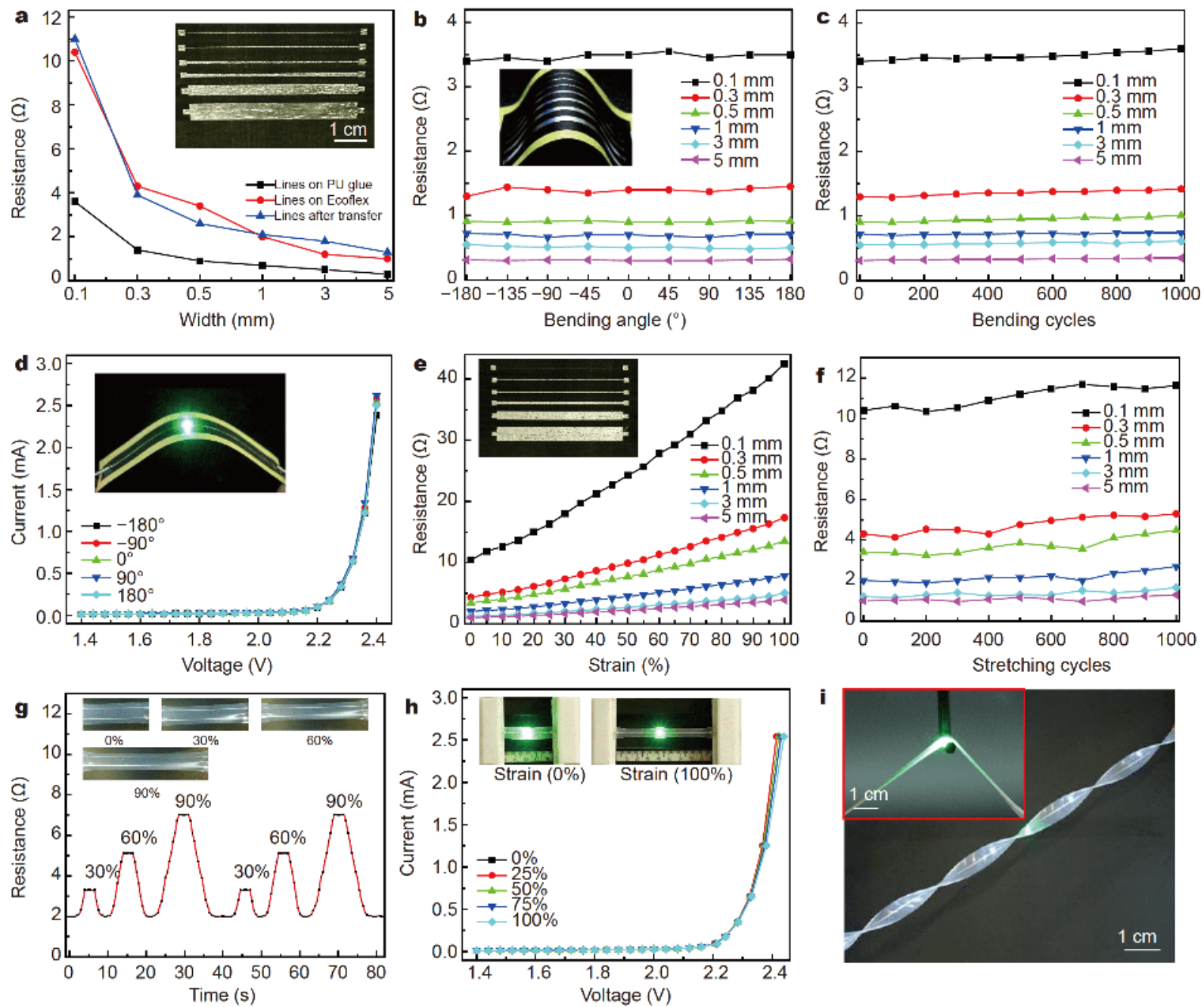

Figure 4 The electrical tests of the Ni-EGaIn lines printed on thermal transfer paper and the stretchable lines transfer printed on ecoflex. (a) The resistance of Ni-EGaIn lines with different widths (length of $5 \mathrm{~cm}$, width of $0.1,0.3,0.5,1,3$, and $5 \mathrm{~mm}$, respectively). (b) The resistance of Ni-EGaIn lines with different widths during the bending process (bending angle from $-180^{\circ}$ to $180^{\circ}$ ). (c) The relationship between bending cycles and the resistances of Ni-EGaIn lines with different widths. (d) The $I-V$ curves of the Ni-EGaIn lines with LED under different bending conditions (bending angle from $-180^{\circ}$ to $180^{\circ}$ ). (e) The resistance of Ni-EGaIn lines with different widths under various strains. (f) The relationship between stretching cycles and the resistances of Ni-EGaIn lines with different widths. (g) The resistance curve of the Ni-EGaIn lines stretched to different lengths (30\%, $60 \%$ and $90 \%$ ). (h) The $I-V$ curves of the Ni-EGaIn lines with LED under various strains (strain $0 \%$ to $100 \%$ ). (i) The photographs of the LED lights packaged in ecoflex stretched vertically and distorted.

chip resistor (length of $1.4 \mathrm{~mm}$, width of $0.73 \mathrm{~mm}$ and height of $1 \mathrm{~mm}$ ) is attached to the Ni-EGaIn line (Fig. 3g). Due to the favorable wettability of Ni-EGaIn on tin plated pins, the two ends of this component infiltrate into the Ni-EGaIn, demonstrating the fine connection between Ni-EGaIn and the chip components. Fig. $3 \mathrm{~h}$ shows the folded thermal transfer paper (bending angle of $180^{\circ}$ ) and the bending part. The printed $\mathrm{Ni}$ EGaIn adheres to the PU glue closely and maintains stable surface topography.

The Ni-EGaIn lines printed on the thermal transfer paper possess high stability and good conductivity. Fig. 4a shows the resistance of Ni-EGaIn lines with different widths (length of $5 \mathrm{~cm}$, width of $0.1,0.3,0.5,1,3$ and $5 \mathrm{~mm}$, respectively) printed on the PU glue and transferred on ecoflex. In particular, the remained resistances of Ni-EGaIn lines on the PU glue after transfer printing were also measured (the blue curve). The resistance increased gradually with the decrease of the line width. The maximum resistance was $3.6 \Omega$ with a line width of $0.1 \mathrm{~mm}$, and the minimum resistance was $0.3 \Omega$ with a line width of $5 \mathrm{~mm}$. The resistance of 
metallic conductors can be calculated by the equation: $R=\rho L / S$, where $R$ is the resistance, $\rho$ is the resistivity, $L$ is the length of the conductor, and $S$ is the cross-sectional area of the conductor. Here, the conductivity $(1 / \rho)$ of NiEGaIn is about $2.01 \times 10^{6} \mathrm{~S} \mathrm{~m}^{-1}$. The theoretical resistance of the $0.1-\mathrm{mm} \mathrm{Ni-EGaIn} \mathrm{line} \mathrm{is} 50$ times higher than that of the 5 -mm Ni-EGaIn line, while the actual resistance is about 12 times higher. This result suggests that the crosssectional area of Ni-EGaIn lines is uneven, consistent with the SEM results in Fig. 3e, f. Thus, it is of great importance to measure the resistance of the Ni-EGaIn lines before practical applications. The resistance of transfer-printed Ni-EGaIn lines on ecoflex is significantly higher than that of the original Ni-EGaIn lines rolling printed on PU glue (the maximum resistance is $10.4 \Omega$ with a line width of $0.1 \mathrm{~mm}$, and the minimum resistance is $1.0 \Omega$ with a line width of $5 \mathrm{~mm}$ ), and the remained $\mathrm{Ni}$ EGaIn lines on PU glue also show higher resistance than the original Ni-EGaIn lines (the maximum resistance is $11.0 \Omega$ with line width of $0.1 \mathrm{~mm}$, and the minimum resistance is $1.3 \Omega$ with line width of $5 \mathrm{~mm}$ ). These results indicate that only a part of Ni-EGaIn is transferred to ecoflex, reducing the cross area of the Ni-EGaIn lines. Besides, the Ni-EGaIn would be oxidized and covered with a layer of oxide during the transfer printing process, which would reduce the electrical conductivity of $\mathrm{Ni}$ EGaIn. The schematic diagram in Fig. S4 shows the formation of oxide during the transfer printing process.

The thermal transfer paper with excellent flexibility can be bent to change morphology, as shown in Fig. 4b. We first evaluated the resistance of these Ni-EGaIn lines with different widths during the bending process (bending angle from $-180^{\circ}$ to $180^{\circ}$ ). In Fig. $4 \mathrm{~b}$, the resistance of the bent $\mathrm{Ni}$-EGaIn lines shows slight variation during bending $(\Delta R=0.15 \Omega$ of $0.1 \mathrm{~mm}$ lines, $\Delta R=0.15 \Omega$ of $0.3 \mathrm{~mm}$ lines, $\Delta R=0.02 \Omega$ of $0.5 \mathrm{~mm}$ lines, $\Delta R=0.06 \Omega$ of $1 \mathrm{~mm}$ lines, $\Delta R=0.07 \Omega$ of $3 \mathrm{~mm}$ lines, $\Delta R=0.02 \Omega$ of $5 \mathrm{~mm}$ lines, respectively). Furthermore, 1,000 repetitive bending cycles in the backward direction to $180^{\circ}$ do not induce noticeable change in the resistance of the $\mathrm{Ni}$ EGaIn lines (Fig. 4c), indicating excellent electrical stability. We connected the Ni-EGaIn lines (width of $1 \mathrm{~mm}$ ) to an LED light, and tested the $I-V$ (currentvoltage) curves of the Ni-EGaIn lines with LED under different bending conditions (bending angle from $-180^{\circ}$ to $180^{\circ}$ ), as shown in Fig. $4 \mathrm{~d}$. The maximum resistance variation ratio of the Ni-EGaIn lines is about $8 \%$, which is rather smaller than that of the LED light (above $3 \mathrm{k} \Omega$ ), demonstrating the resistance variation of the Ni-EGaIn lines has slight influence on the electronic components and the whole electric circuits.

To assess its potential as stretchable conductive materials, the Ni-EGaIn lines transfer printed on ecoflex were further subjected to a series of electrical tests. Fig. $4 \mathrm{e}$ depicted the resistance of Ni-EGaIn lines with different widths under various strains. All the resistances of $\mathrm{Ni}$ EGaIn lines gradually increased with the stretch length, and no rupture of the Ni-EGaIn lines was noticed even under large strains up to $100 \%$. Here, the cross-sectional area $(S)$ of the Ni-EGaIn line and its length $(L)$ follow the equation: $S=V / L$, where $V$ is the volume of Ni-EGaIn line. The volume is constant under various strains $\left(V=V_{0}\right)$. Therefore, the relationship between the tensile elongation and resistance variation of the Ni-EGaIn line can be calculated by the following equation,

$$
\frac{R}{R_{0}}=\frac{\frac{\rho \cdot L}{S}}{\frac{\rho \cdot L_{0}}{S_{0}}}=\frac{V_{0}}{V}\left(\frac{L}{L_{0}}\right)^{2}=\left(\frac{L}{L_{0}}\right)^{2},
$$

where, $R_{0}, L_{0}, S_{0}$ and $V_{0}$ are the resistance, length, crosssectional area and volume of Ni-EGaIn line under $0 \%$ strain, respectively. The quadratic functions were selected to fit the curves. For example, the fitting function $\left(R^{2}=0.9993\right)$ of the resistance curve of Ni-EGaIn line width of $0.1 \mathrm{~mm}$ is shown as the following equation:

$R=0.0008 x^{2}+0.2385 x+10.195$,

where, $R$ is the resistance $(\Omega)$ of Ni-EGaIn line, and $x$ is the strain (\%) of Ni-EGaIn line. This result suggests that the Ni-EGaIn lines on ecoflex show great potential as resistance variable strain sensors. Furthermore, for practical applications of strain sensors, long-term stability and durability is of great importance. The Ni-EGaIn lines exhibit good long-term stability during 1,000 loading and unloading cycles of $50 \%$ strain (Fig. $4 \mathrm{f}$ ). However, there is a slight increase of the resistance of all the Ni-EGaIn lines on ecoflex after 1,000 cycles, which is related to the nonreversible deformation of ecoflex. Furthermore, the NiEGaIn line (width of $1 \mathrm{~mm}$ ) was stretched to different lengths $(30 \%, 60 \%$ and $90 \%)$ for two cycles, and the curve showed that the Ni-EGaIn line possessed rather high stability (Fig. 4g). In addition, a LED light was attached to the ecoflex and connected with the Ni-EGaIn line (width of $1 \mathrm{~mm}$ ), as shown in Fig. $4 \mathrm{~h}$. The $I-V$ curves of the NiEGaIn lines with LED under various strains ( $0 \%$ to $100 \%)$ show that the Ni-EGaIn lines have remarkable stability and do not affect the normal operation of the circuits. Although the LED light packaged in ecoflex was stretched vertically and further distorted, the whole electric circuit maintains normal work, as shown in Fig. 4i. 
a

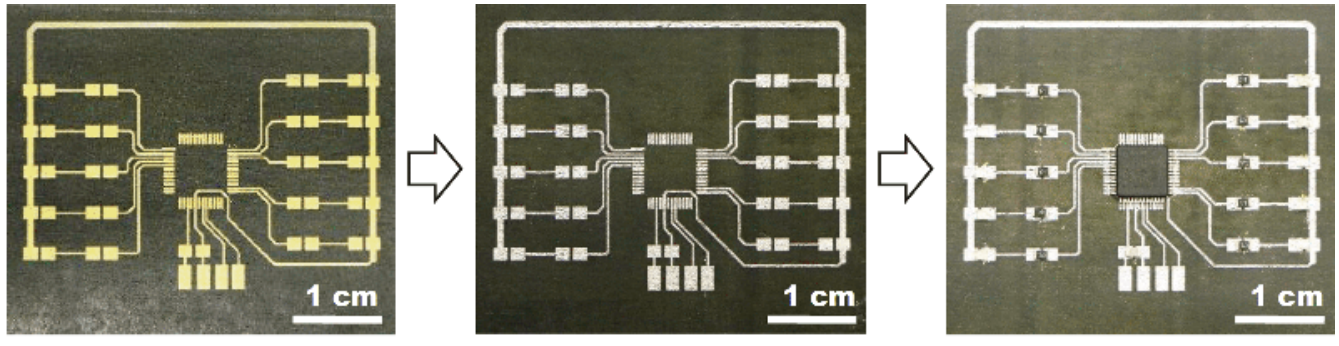

b
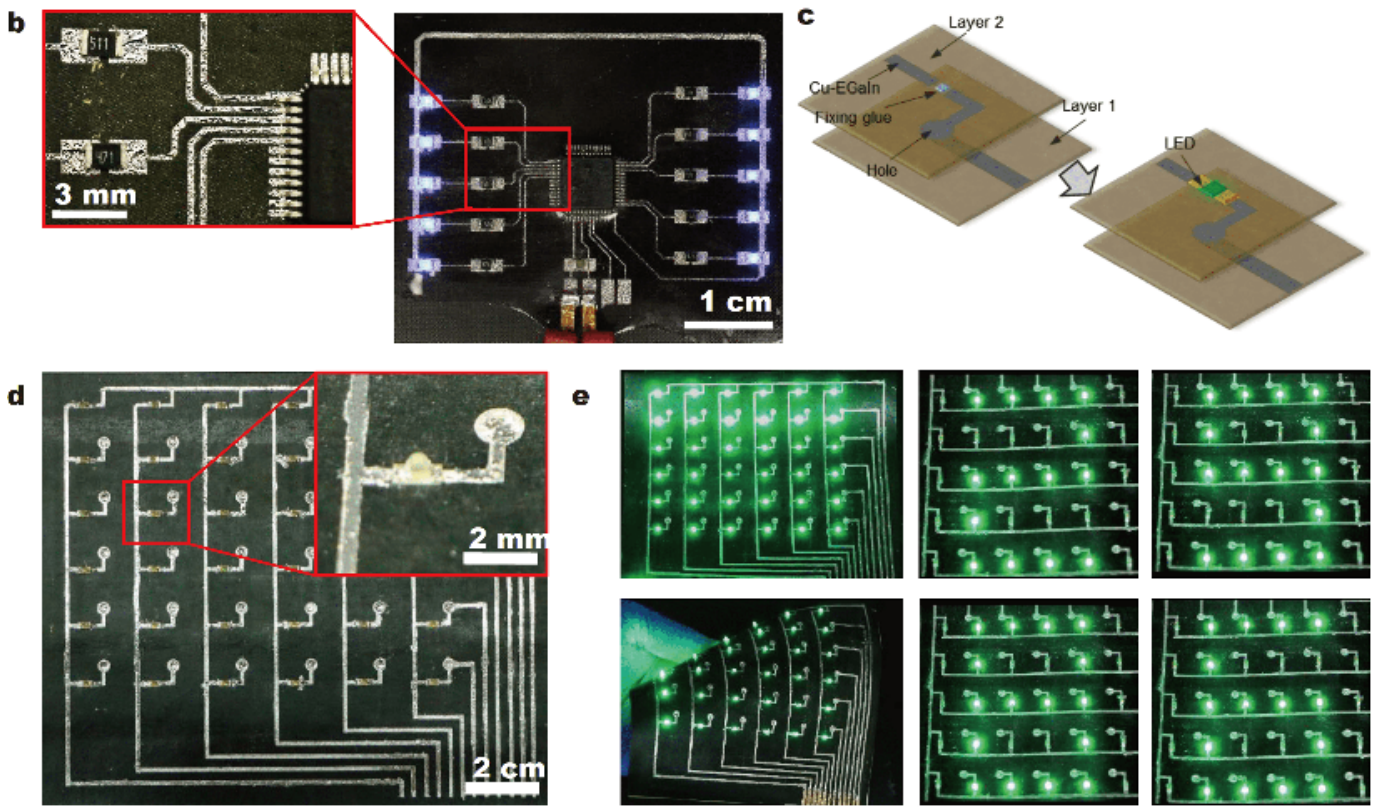

Figure 5 The applications of flexible electronics based on rolling printing method. (a) The LED array flexible paper circuit. (b) The detail and working pictures of the LED array flexible paper circuit. (c) The schematic diagram about the connection of Ni-EGaIn on two paper layers. (d) The photographs of the double layer paper circuit. (e) The double layer paper LEDs array served as display.

In addition, to demonstrate the excellent and stable electrical properties of the flexible paper electronics based on rolling printing method, an LED array flexible paper circuit was fabricated, as shown in Fig. 5a. An MCU (STC $15 \mathrm{~W} 4 \mathrm{~K} 56 \mathrm{~S} 4$ ) was selected to control the LED lights on in turn, followed by reciprocating, as shown in Fig. 5b. The preparation method of paper electronics also provided a straightforward process to fabricate multilayer circuits and large area conductive patterns.

As shown in Fig. 5c-e, we fabricated a double layer paper circuit, serving as a $6 \times 6$ LEDs array. Fig. $5 c$ shows the connection of Ni-EGaIn on two paper layers. Fig. S5 shows the preparation of the double layer paper circuit. At first, the Ni-EGaIn was rolling printed on the double layers paper. Then, a $6 \times 6$ holes array was made on the second layer paper. Later, these two layers were glued together by fixing glue, and a little of Ni-EGaIn was filled into the holes. The Ni-EGaIn lines on two layers were thus connected together. Finally, the LED lights were adhered to the second layer paper by fixing glue. In addition, a series of large area conductive patterns were fabricated by this rolling printing method (Fig. 6a, b and Fig. S6). In particular, the Ni-EGaIn could be rolling printed on colored toners, as shown in Fig. 6c. Besides, a Chinese calligraphy $(15 \mathrm{~cm} \times 28 \mathrm{~cm})$ also could be rolling printed, as shown in Fig. $6 \mathrm{~d}$.

We fabricated a stretchable LED array circuit, as shown in Fig. 7a. This LED array circuit could be stretched to $100 \%$ strain and maintained normal work, proving the promising potential and usefulness of SMART method in quickly making wearable electronics. To verify the cyclic stability of this device, the LED array circuit was stretched to $100 \%$ strain and twisted for 100 times, which also maintained normal work, as shown in Fig. S7.

Fig. S8 shows the preparation process of the LED array circuit. At first, the Ni-EGaIn was rolling printed on the paper, then transfer-printed on ecoflex. Later, the LEDs were connected with the Ni-EGaIn lines and fixed using 

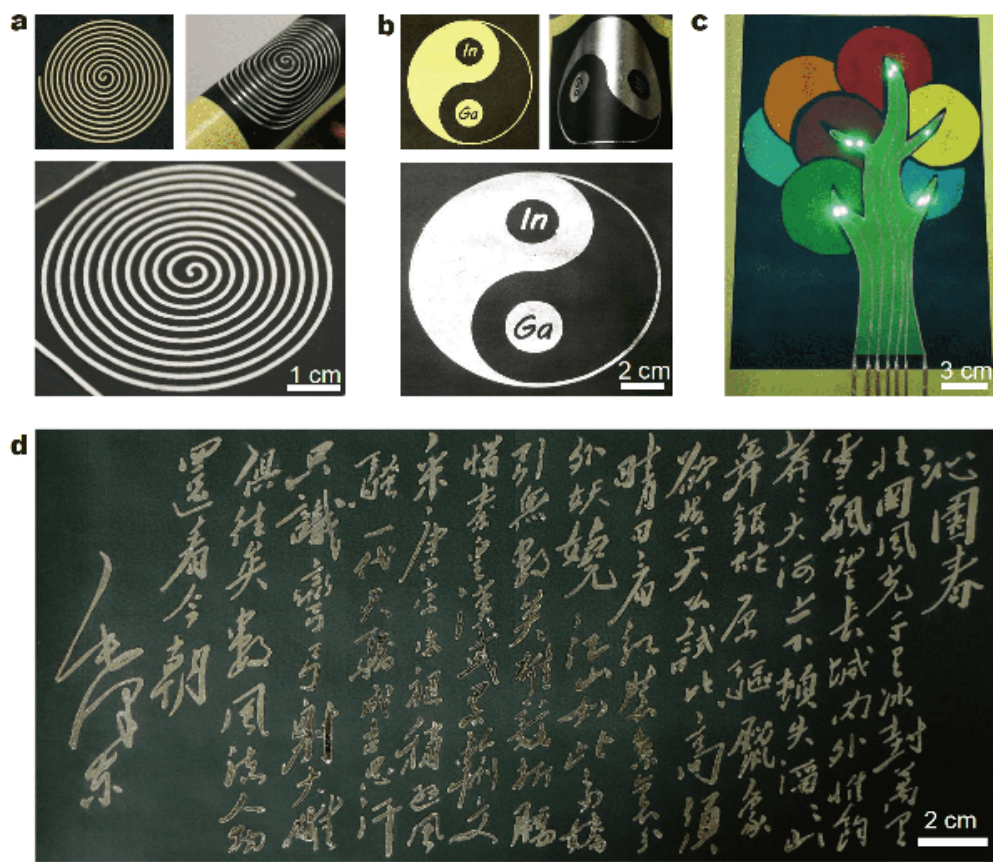

Figure 6 A series of large area conductive patterns on paper. (a) The large area solenoid coil patterns. (b) The conductive Taiji patterns. (c) The NiEGaIn rolling printed on paper with colored toners. (d) The large area Chinese calligraphy conductive pattern.

the fixing glue. Meanwhile, two $\mathrm{Cu}$ wires were inserted into the ends of Ni-EGaIn lines to provide a voltage of 3.3 V. Finally, some mixed Ecoflex 00-30 precursors were poured on the transfer-printed Ni-EGaIn to pack it. The transfer-printed Ni-EGaIn lines on ecoflex could be directly assembled on human bodies to monitor the motion of joints because of its high stretchability and biocompability. Here, we designed a Ni-EGaIn strain sensor (line width of $1 \mathrm{~mm}$ ) and tested its resistance under various strains, as shown in Fig. 7b. Fig. 59 shows the preparation process of the Ni-EGaIn strain sensor, similar to the LED array circuit. The resistance of this strain sensor had an obvious change from $0 \%$ strain to $100 \%$ strain.

The Ni-EGaIn strain sensor was attached to a wrist by medical adhesive tape to monitor the motion of the wrist joints. Fig. $7 \mathrm{c}$ shows the resistance of this strain sensor under various bending angles of the wrist joints. The bending angles of the wrist joints could be calculated by measuring the resistance of this strain sensor in practical application. Similarly, a repeatable response of the $\mathrm{Ni}$ EGaIn strain sensor to the motion of a wrist joint also was verified (Fig. 7d).

Furthermore we found that the Ni-EGaIn printed on PU glue could be removed by alcoholic solution, as shown in Fig. 8a, b. Sprayed with the alcoholic solution and using a brush, the Ni-EGaIn could be removed and gathered into a droplet (Fig. 8b). Here, the weight of the original printed $\mathrm{Ni}$-EGaIn was about $0.3 \mathrm{~g}$, and the remained Ni-EGaIn on the paper was about $0.1 \mathrm{mg}$. This discovery could be used to recycle Ni-EGaIn so as to decrease the fabrication cost and reduce environmental pollution. In addition, the Ni-EGaIn lines printed on paper could also be burnt as biodegradable wastes, as shown in Fig. 8c.

Overall, typical merits of the semi-liquid metal rolling and transfer printing method for making flexible electronics can be summarized as follows: (i) super-fast fabrication for paper and stretchable electronics with printing time of $10 \mathrm{~s}$, (ii) excellent electrical stability of Ni-EGaIn lines on paper and ecoflex, (iii) an simple method for the preparation of multilayer and large area circuits, (iv) recyclable ability to further reduce the cost of fabrication and environment pollution.

\section{CONCLUSIONS}

In summary, we established a super-fast and low-cost process-SMART printing method to fabricate flexible electronics on paper and ecoflex substrates by rolling and transfer printing the semi-liquid metal (Ni-EGaIn). The interface adhesion-selection principle between Ni-EGaIn and different target substrates (toners, PU glue and 

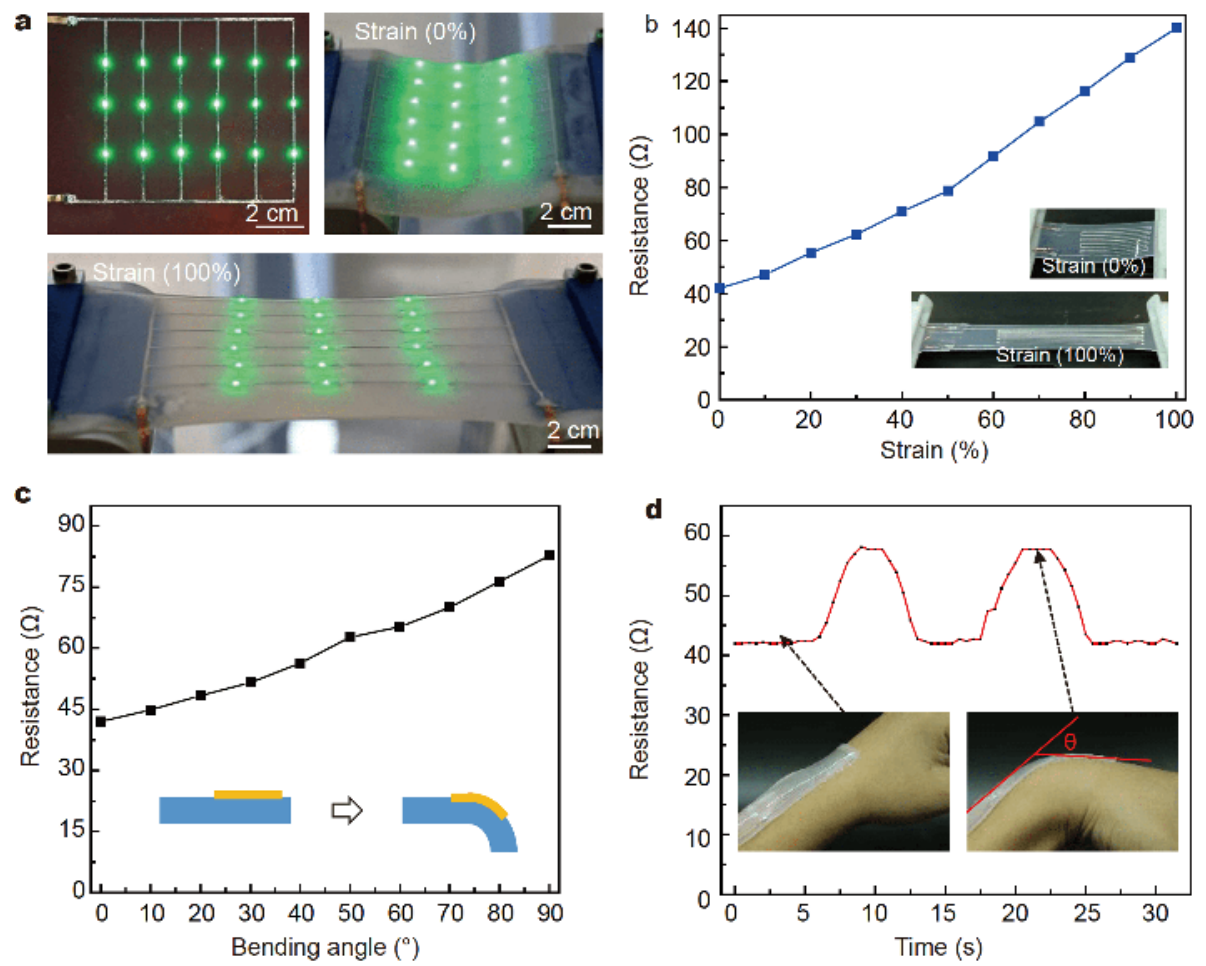

Figure 7 (a) The photographs of the stretchable LED array circuit. (b) The resistance of the Ni-EGaIn strain sensor under various strains. (c) The resistance of this strain sensor under various bending angles of the wrist joints. (d) Responsive signal of Ni-EGaIn strain sensor during cyclic bendingunbending motion of the wrist.

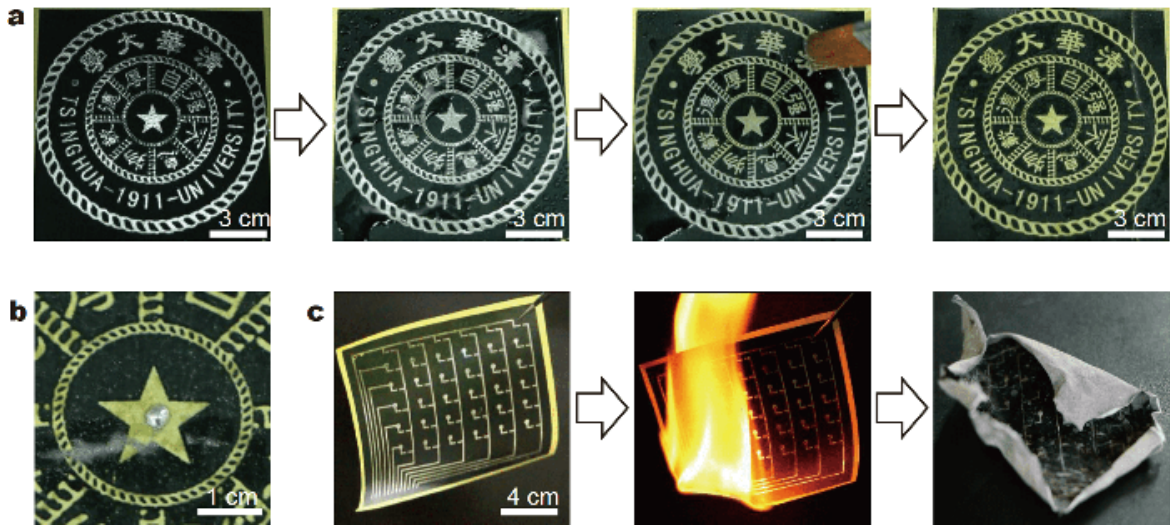

Figure 8 (a) The photographs of the recycle process of Ni-EGaIn printed on PU glue. (b) The photograph of the gathered Ni-EGaIn droplet. (c) The photographs of the burnt process of Ni-EGaIn.

ecoflex) also was disclosed. The conceptual experiments elucidated that the paper electronics had excellent electrical stability and durability after repeated cycles of more than 1,000 times and the stretchable electronics on ecoflex could be stretched to $100 \%$ strain. Besides, we demonstrated the capability of the SMART printing method in the field of wearable electronics, multilayer and large area circuits. With these important features, SMART printing method is a potential mass production way to quickly manufacture various functional flexible electronics in the coming time.

Received 10 December 2018; accepted 29 January 2019; published online 27 February 2019 
1 Lou Z, Chen S, Wang L, et al. Ultrasensitive and ultraflexible eskins with dual functionalities for wearable electronics. Nano Energy, 2017, 38: 28-35

2 Khan Y, Ostfeld AE, Lochner CM, et al. Monitoring of vital signs with flexible and wearable medical devices. Adv Mater, 2016, 28: 4373-4395

3 Hammock ML, Chortos A, Tee BCK, et al. 25th anniversary article: the evolution of electronic skin (e-skin): a brief history, design considerations, and recent progress. Adv Mater, 2013, 25: 59976038

4 Wang L, Jackman JA, Ng WB, et al. Flexible, graphene-coated biocomposite for highly sensitive, real-time molecular detection. Adv Funct Mater, 2016, 26: 8623-8630

5 Wang L, Chen D, Jiang K, et al. New insights and perspectives into biological materials for flexible electronics. Chem Soc Rev, 2017, 46: 6764-6815

6 Kaltenbrunner M, Sekitani T, Reeder J, et al. An ultra-lightweight design for imperceptible plastic electronics. Nature, 2013, 499: 458-463

7 Kim CC, Lee $\mathrm{HH}$, Oh $\mathrm{KH}$, et al. Highly stretchable, transparent ionic touch panel. Science, 2016, 353: 682-687

8 Lee J, Kwon H, Seo J, et al. Conductive fiber-based ultrasensitive textile pressure sensor for wearable electronics. Adv Mater, 2015, 27: 2433-2439

9 Chou HH, Nguyen A, Chortos A, et al. A chameleon-inspired stretchable electronic skin with interactive colour changing controlled by tactile sensing. Nat Commun, 2015, 6: 8011

10 Rogers JA, Someya T, Huang Y. Materials and mechanics for stretchable electronics. Science, 2010, 327: 1603-1607

11 Webb RC, Bonifas AP, Behnaz A, et al. Ultrathin conformal devices for precise and continuous thermal characterization of human skin. Nat Mater, 2013, 12: 938-944

12 Liu J, Yi L. Liquid Metal Biomaterials: Principles and Applications. Singapore: Springer, 2018

13 Xu R, Lee JW, Pan T, et al. Designing thin, ultrastretchable electronics with stacked circuits and elastomeric encapsulation materials. Adv Funct Mater, 2017, 27: 1604545

14 Kim BH, Kim JH, Persano L, et al. Dry transient electronic systems by use of materials that sublime. Adv Funct Mater, 2017, 27: 1606008

15 Fang H, Yu KJ, Gloschat C, et al. Capacitively coupled arrays of multiplexed flexible silicon transistors for long-term cardiac electrophysiology. Nat Biomed Eng, 2017, 1: 0038

16 Wang Y, Zhu C, Pfattner R, et al. A highly stretchable, transparent, and conductive polymer. Sci Adv, 2017, 3: e1602076

17 Savagatrup S, Chan E, Renteria-Garcia SM, et al. Plasticization of PEDOT:PSS by common additives for mechanically robust organic solar cells and wearable sensors. Adv Funct Mater, 2015, 25: 427436

18 Lipomi DJ, Vosgueritchian M, Tee BCK, et al. Skin-like pressure and strain sensors based on transparent elastic films of carbon nanotubes. Nat Nanotech, 2011, 6: 788-792

19 Oh JY, Kim S, Baik HK, et al. Conducting polymer dough for deformable electronics. Adv Mater, 2016, 28: 4455-4461

20 Tang SY, Sivan V, Petersen P, et al. Liquid metal actuator for inducing chaotic advection. Adv Funct Mater, 2014, 24: 5851-5858

21 Gao Y, Li H, Liu J. Directly writing resistor, inductor and capacitor to composite functional circuits: A super-simple way for alternative electronics. PLoS ONE, 2013, 8: e69761

22 Wang X, Zhang Y, Guo R, et al. Conformable liquid metal printed epidermal electronics for smart physiological monitoring and simulation treatment. J Micromech Microeng, 2018, 28: 034003

23 Wang X, Yao W, Guo R, et al. Soft and moldable Mg-doped liquid metal for conformable skin tumor photothermal therapy. Adv Healthcare Mater, 2018, 7: 1800318

24 Yi L, Jin C, Wang L, et al. Liquid-solid phase transition alloy as reversible and rapid molding bone cement. Biomaterials, 2014, 35 9789-9801

25 Varga M, Mehmann A, Marjanovic J, et al. Adsorbed eutectic GaIn structures on a neoprene foam for stretchable MRI coils. Adv Mater, 2017, 29: 1703744

26 Tang W, Jiang T, Fan FR, et al. Liquid-metal electrode for highperformance triboelectric nanogenerator at an instantaneous energy conversion efficiency of $70.6 \%$. Adv Funct Mater, 2015, 25: 3718-3725

27 Guo R, Wang X, Chang $\mathrm{H}$, et al. Ni-GaIn amalgams enabled rapid and customizable fabrication of wearable and wireless healthcare electronics. Adv Eng Mater, 2018, 20: 1800054

28 Guo R, Sheng L, Gong HY, et al. Liquid metal spiral coil enabled soft electromagnetic actuator. Sci China Technol Sci, 2018, 61: 516-521

29 Jin SW, Park J, Hong SY, et al. Stretchable loudspeaker using liquid metal microchannel. Sci Rep, 2015, 5: 11695

30 Kubo M, Li X, Kim C, et al. Stretchable microfluidic radiofrequency antennas. Adv Mater, 2010, 22: 2749-2752

31 Guo C, Yu Y, Liu J. Rapidly patterning conductive components on skin substrates as physiological testing devices via liquid metal spraying and pre-designed mask. J Mater Chem B, 2014, 2: 57395745

32 Guo R, Wang XL, Yu WZ, et al. A highly conductive and stretchable wearable liquid metal electronic skin for long-term conformable health monitoring. Sci China Technol Sci, 2018, 61: 1031-1037

33 Wang L, Liu J. Pressured liquid metal screen printing for rapid manufacture of high resolution electronic patterns. RSC Adv, 2015, 5: 57686-57691

34 Zhang Q, Zheng Y, Liu J. Direct writing of electronics based on alloy and metal (DREAM) ink: A newly emerging area and its impact on energy, environment and health sciences. Front Energy, 2012, 6: 311-340

35 Wang Q, Yu Y, Yang J, et al. Fast fabrication of flexible functional circuits based on liquid metal dual-trans printing. Adv Mater, 2015, 27: 7109-7116

36 Blanco MB, Dausmann KH, Ranaivoarisoa JF, et al. Underground hibernation in a primate. Sci Rep, 2013, 3: 1768

37 Zheng Y, He ZZ, Yang J, et al. Personal electronics printing via tapping mode composite liquid metal ink delivery and adhesion mechanism. Sci Rep, 2015, 4: 4588

38 Lu T, Finkenauer L, Wissman J, et al. Rapid prototyping for softmatter electronics. Adv Funct Mater, 2014, 24: 3351-3356

39 Jeong YR, Kim J, Xie Z, et al. A skin-attachable, stretchable integrated system based on liquid GaInSn for wireless human motion monitoring with multi-site sensing capabilities. NPG Asia Mater, 2017, 9: e443

40 Mohammed MG, Kramer R. All-printed flexible and stretchable electronics. Adv Mater, 2017, 29: 1604965

41 Tang J, Zhao X, Li J, et al. Gallium-based liquid metal amalgams: transitional-state metallic mixtures (trans $\mathrm{M}^{2}$ ixes) with enhanced and tunable electrical, thermal, and mechanical properties. ACS Appl Mater Interfaces, 2017, 9: 35977-35987 
Chang H, Guo R, Sun Z, et al. Direct writing and repairable paper flexible electronics using nickel-liquid metal ink. Adv Mater Interfaces, 2018, 5: 1800571

43 Yun J, Lim Y, Lee $\mathrm{H}$, et al. A patterned graphene/ZnO UV Sensor driven by integrated asymmetric micro-supercapacitors on a liquid metal patterned foldable paper. Adv Funct Mater, 2017, 27: 1700135

44 Li G, Lee DW. An advanced selective liquid-metal plating technique for stretchable biosensor applications. Lab Chip, 2017, 17: 3415-3421

45 Guo R, Tang J, Dong S, et al. One-step liquid metal transfer printing: toward fabrication of flexible electronics on wide range of substrates. Adv Mater Technol, 2018, 3: 1800265

46 Zhang Q, Gao Y, Liu J. Atomized spraying of liquid metal droplets on desired substrate surfaces as a generalized way for ubiquitous printed electronics. Appl Phys A, 2014, 116: 1091-1097

47 Chrimes AF, Berean KJ, Mitchell A, et al. Controlled electrochemical deformation of liquid-phase gallium. ACS Appl Mater
Interfaces, 2016, 8: 3833-3839

Acknowledgements This work is partially supported by the National Natural Science Foundation of China Key Project (91748206) and Dean's Research Funding and the Frontier Project of the Chinese Academy of Sciences.

Author contributions Guo R and Liu J conceived this work and wrote the article. Guo R performed all the experiments and interpreted the results. All authors joined the research and discussed the results. Liu J supervised the work.

Conflict of interest The authors declare no conflict of interest in this research.

Supplementary information Supporting data are available in the online version of the paper.

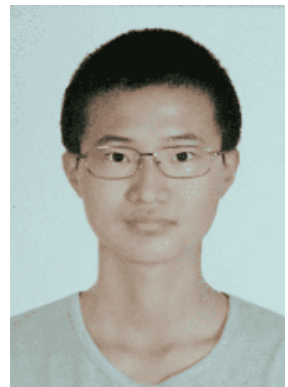

Rui Guo received his BE degree in biomedical engineering from Tsinghua University in 2016. Now he is a PhD candidate majored in biomedical engineering in Tsinghua University. His interest focuses on gallium-based liquid metal flexible and wearable electronics.

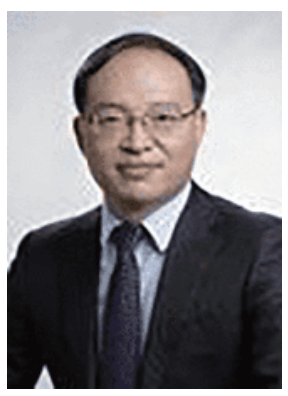

Jing Liu is a jointly appointed professor of Tsinghua University and Technical Institute of Physics and Chemistry, Chinese Academy of Sciences. He also performs visiting research at both Purdue University and Massachusetts Institute of Technology. His research on liquid metal soft machines, biomaterials, printed electronics, and chip cooling has initiated many game changing technologies.

\section{基于半液态金属选择性黍附机理的滚动涂覆与转印技术(SMART): 普适性柔性电子器件快速制备} 国瑞 ${ }^{1}$, 姚思远 ${ }^{2}$, 孙旭阳 ${ }^{2}$, 刘静 ${ }^{1,2^{*}}$

摘要 近年来液态金属研究取得了突破性进展, 其在现代柔性电子领域显示出巨大的应用前景. 本文基于半液态金属墨水及其在不同基 底表面的选择性粘附机理，提出了一种具有普适性的柔性电子超快速制造技术. 该技术成本低，可用于制备大面积高精度液态金属线路， 且制造速率远远超过经典电子制造及液态金属电路打印技术. 本文制备了一系列具有优良的电学稳定性和适应性的柔性、可拉伸电路, 如多层电路、大面积电路以及拉伸传感器等. 此外, 基于该技术制备的液态金属电路具有可回收的优点. 该技术的实施无需复杂设备, 有 望在今后的工业生产和个人消费电子领域发挥重要作用. 